\title{
Sleep-Wake Abnormalities in Patients with Cirrhosis
}

\author{
Sara Montagnese*, Cristiano De Pittà ${ }^{\#}$, Michele De Rui*, Michela Corrias*, Matteo Turco*, \\ Carlo Merkel*, Piero Amodio*, Rodolfo Costa ${ }^{\#}$, Debra J. Skene ${ }^{\circ}$, Angelo Gatta*
}

*Department of Medicine, University of Padova, Italy

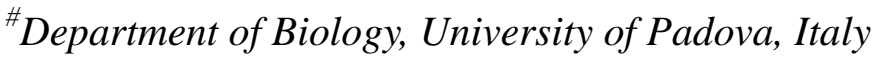

${ }^{\circ}$ Faculty of Health and Medical Sciences, University of Surrey, Guildford, United Kingdom

\section{For correspondence:}

Sara Montagnese

Dipartimento di Medicina

Via Giustiniani, 2

35128 Padova, Italia

Tel +390498218675

Fax +390497960903

sara.montagnese@unipd.it

Word Count: 4818 (with references) 
A considerable proportion of patients with cirrhosis exhibit disturbed night sleep, delayed sleep habits and excessive daytime sleepiness. These have been variously attributed to hepatic encephalopathy and impaired hepatic melatonin metabolism, but the understanding of their pathophysiology remains limited and their treatment problematic. Sleep is regulated by the interaction of a homeostatic and a circadian process. The homeostatic process determines sleep propensity in relation to sleep-wake history, thus the need to sleep increases with the duration of the waking period. The circadian process, which is marked by the 24-hour rhythm of the hormone melatonin, is responsible for the alternation of high/low sleep propensity in relation to dark/light cues. Circadian sleep regulation has been studied in some depth in patients with cirrhosis, who show delays in the 24 -hour melatonin rhythm, most likely in relation to reduced sensitivity to light cues. However, while melatonin abnormalities are associated with delayed sleep habits, they do not seem to offer a comprehensive explanation to the disturbances in night sleep exhibited by these patients. Fewer data are available on homeostatic sleep control: it has been recently hypothesised that patients with cirrhosis and hepatic encephalopathy might be unable, due to excessive daytime sleepiness, to accumulate the need/ability to produce restorative sleep. This review will describe in some detail the features of sleep-wake disturbances in patients with cirrhosis, their mutual relationships and those, if any, with hepatic failure/hepatic encephalopathy. A separate section will cover the available information on their pathophysiology. Finally, aetiological treatment will be briefly discussed. 


\section{Sleep-wake disturbances and their assessment}

Approximately $10 \%$ of an otherwise healthy population suffers from a clinically significant sleep-wake disorder (1). Sleep-wake disorders are also associated with medical conditions such as cardiovascular disease, diabetes and depression. Difficulties sleeping can depend on the pain/discomfort associated with a particular disease, or can be a direct consequence of its pathophysiology. The majority of individuals reporting sleep-wake disturbance present with one or more of the so called 'classical complaints': i) insomnia, or a difficulty attaining and/or sustaining sleep; ii) hypersomnia or excessive sleepiness, which is the propensity to sleep at inappropriate times; iii) unusual events associated with sleep, such as heavy snoring, apnoea or limb movements. Sleep-wake evaluation begins with the definition of the complaint, together with a patient profile, including age, occupational/marital status and living arrangements. Details concerning the sleep problem are obtained, either by structured interviews or specific, validated questionnaires. The patient's habitual daily schedule is reviewed, preferably by use of sleep diaries, over a period of 2-3 weeks. Laboratory-based nocturnal polysomnography is considered a gold standard but is expensive, time-consuming and thus cannot be used for first-line assessment purposes. Wrist actigraphy is a 'lighter' technique, based on the registration of movement by means of an accelerometer, and on the assumption: sleep = no movement. An actigraph looks like a wristwatch and can be worn for days/weeks, without interfering with normal life, to assess rest-activity patterns. In recent years, it has also become common to assess a subject's natural propensity to be more of a morning (lark) or an evening (owl) person/type; this is referred to as chronotype or diurnal preference.

\section{Sleep-wake disturbances in patients with cirrhosis}

So-called 'sleep-wake inversion', or the combination of restless nights and excessive daytime sleepiness, was first recognised as a sign of overt hepatic encephalopathy (HE) by Sherlock and 
colleagues (2). There is also anecdotal evidence that sleep abnormalities worsen following the insertion of transjugular intra-hepatic portal-systemic shunts (3), and improve after initiation of treatment with lactulose (4). These findings have lead to the idea that the pathogenesis of sleep-wake disturbances in patients with cirrhosis is closely related to that of HE, and that sleep and neuropsychiatric abnormalities are invariably associated in these patients. There is limited evidence, however, to support this theory, especially in human studies. In addition, lack of sleep per se can result in neuropsychological abnormalities, thus potentially confounding the diagnosis of minimal/mild HE. Finally, limited attempts have been made to characterise sleep-wake abnormalities in patients with cirrhosis. With notable exceptions (5), reports have often been based on responses to unspecific sleep measures derived from quality of life or psychological well-being questionnaires (6). In this review, we attempt to approach sleep-wake disturbances in patients with cirrhosis in a descriptive fashion, without a predefined interpretative frame. The available evidence on their pathophysiology is also examined.

\section{Insomnia}

Insomnia seems to be considerably more common in patients with cirrhosis than in patients with other chronic illnesses, for example renal failure (5). Insomnia is also detected in well-compensated cirrhotics $(5,7)$, with no obvious reasons for disturbed sleep such as itching, tense ascites or treatment with diuretics. Up to 50-65\% of patients with cirrhosis complain of unsatisfactory night sleep, mostly in relation to difficulties falling asleep and multiple night awakenings (5-8). Questionnaire-based sleep complaints have been substantiated by wrist actigraphy, which documents activity over the whole 24hours, and frequent sleep interruptions (5,8-10). Insomnia has been traditionally associated with HE, and there are some experimental animal data to support this association (11-13). Regarding human studies, Córdoba and co-workers found no difference in the prevalence of insomnia in relation to psychometric performance in 44 patients with cirrhosis, 24 of whom had minimal HE (5). Montagnese and co-workers also observed no association between the presence of insomnia and the 
presence/severity of $\mathrm{HE}$ in 87 patients, classified as neuropsychiatrically unimpaired or as having minimal/overt HE (7). Thus, based on the current human data, confusion between insomnia and HE should be avoided, especially in relation to the outcomes of treatment trials.

\section{Delayed sleep timing and diurnal preference}

Córdoba and co-workers were the first to assess sleep timing and diurnal preference in patients with cirrhosis, in relation to sleep quality and by comparison with healthy volunteers and patients with renal failure (5). An association was observed between delayed sleep habits/evening preference and impaired sleep quality in patients with cirrhosis, but not in the other two groups. These findings were confirmed by Montagnese and co-workers, who described significant correlations between diurnal preference and sleep quality, with evening type cirrhotic patients taking longer to fall asleep and sleeping worse than their counterparts with earlier sleep timing (7). The observed delays in sleep timing were shown to be independent of employment status in a subsequent, smaller study (9).

It has been demonstrated that even in the healthy population, evening type people can experience serious difficulties when they are required to be operative in the early part of the day (14). Such difficulties, which may translate into morning traffic accidents and poor school/work performances, become particularly prominent in relation to sudden advances of the sleep-wake schedule, such as the spring switch to daylight-saving time or travelling eastwards across time zones (jet-lag East). The 60-minute delay in sleep-wake habits exhibited by patients with cirrhosis is chronic, rather than sudden and externally imposed. However, its effects on health/prognosis are worthy of further study, as circadian disruption caused by experimental, chronic jet-lag or constant exposure to light has been shown to promote the growth of liver tumours in laboratory animals (15).

\section{Excessive daytime sleepiness}

Profound daytime sleepiness was recognised as a sign of overt HE by Sherlock and colleagues in 1954

(2). However, the patient population studied was small and heterogeneous, and the neurological 
abnormalities extremely severe. Excessive daytime sleepiness has been subsequently described in cirrhotic patients with milder neuropsychiatric impairment $(5,7,8)$. In at least two studies, an association between excessive sleepiness and the presence/degree of HE was observed $(7,16)$. In a recent, large study, in which habitual daytime sleepiness was qualified as present/absent, its absence had a negative predictive value of $92 \%$ in relation to the occurrence of HE-related hospitalisations over a follow-up period of 8 months (17). Most convincingly, changes in subjective sleepiness were shown to closely parallel changes in blood ammonia levels in both healthy volunteers and patients with cirrhosis in a study of induced hyperammonaemia (18). These observations fit with the hypothesis that HE may be interpreted, at least to some extent, as a syndrome of decreased vigilance. Indeed, some of its electroencephalographic features, such as the anteriorization of the background alpha rhythm, are reminiscent of those observed during the transition from wake-to-sleep (19). Somewhat counterintuitively, there is limited evidence of a direct relationship between night sleep disturbance and excessive daytime sleepiness in patients with cirrhosis, suggesting that their occurrence may indeed reflect different disease processes (7).

The data presented refer to a sort of 'old-fashioned', archetypical patient with alcohol-related or viral cirrhosis. However, the incidence of non-alcoholic steatohepatitis (NASH)-cirrhosis is increasing dramatically, and given its association with overweight, diabetes, systemic hypertension and obstructive sleep-apnea, a considerably more complex picture will probably need to be built in the future. Evidence in this respect is starting to emerge, for example with: $i$ ) a brief report highlighting an association between loss of slow-wave sleep and reduced levels of ghrelin in a small group of cirrhotic patients with minimal HE (20), and ii) a recent study comparing the polysomnographic characteristics of patients with obstructive sleep-apnea with and without cirrhosis (21).

\section{Physiological sleep-wake regulation}


The currently accepted model of human sleep-wake regulation postulates that two primary components regulate sleep: a circadian process, with near-24-hour periodicity, and a homeostatic process that builds up during wakefulness and declines during sleep (Figure 1A) (22).

\section{Circadian sleep-wake regulation}

Circadian regulation is responsible for the alternation of periods of high/low sleep propensity, in relation to the endogenous timing system and external dark/light cues, and irrespective of preceding sleep-wake behaviour. The suprachiasmatic nuclei (SCN) of the hypothalamus are the site of the socalled master circadian clock, which generates the SCN-driven circadian rhythms of melatonin, cortisol and core body temperature. In humans, the average, endogenous circadian period is slightly longer than 24 hours, and it is constantly synchronised to the 24-hour day by external influences. Light is the major external time cue and it reaches the SCN by afferent projections from the retina, via the retinohypothalamic tract. In turn, the SCN project to the pineal gland via a multisynaptic pathway, regulating the production of melatonin. Melatonin can be thought of as the neuroendocrine transducer of the light/dark cycle. Thus, in healthy individuals, its synthesis increases soon after the onset of darkness, peaks in the middle of the night, and then gradually declines. The nocturnal rise in melatonin synthesis is associated with an increased propensity to sleep and is acutely suppressed by light exposure. Melatonin is mostly hydroxylated and sulphated to 6-sulphatoxymelatonin (aMT6s), primarily via the liver cytochrome CYP1A2 (23), and aMT6s is subsequently excreted in the urine.

\section{The genetics of circadian timing}

The current model for the circadian timing system encompasses a number of clock genes. These encode for proteins directly involved in the molecular mechanism which produces a circadian oscillation. The oscillation is generated autonomously in each of the $\approx 20,000$ neurons clustering within the human SCN (24). Single cell oscillations become synchronised and give rise to a robust, circadian molecular mechanism which acts as a 'master clock'. In turn, this controls the activity of several, so-called clock- 
controlled genes. At the core of the circadian clockwork are a number of overlapping transcriptionaltranslational feedback loops, which comprise positive and negative interconnected arms (25). In a simplified, schematic description of the mammalian circadian machinery, the transcriptional activator proteins BMAL and CLOCK (CLK) form heterodimers which bind to specific elements (E-boxes) located within the promoter regions of the clock genes Period (Per) and Cryptochrome (Cry), and activate their transcription. There are three Per paralogs (Per1, Per2, and Per3) and two Cry paralogs (Cryl and Cry2) in mammalian genomes. The subsequent translation of the corresponding mRNAs into proteins is accompanied by a number of post-translational modifications, which result in a lag of about 4-6 hours between transcription and protein accumulation, and are crucial to sustain rhythmicity. Once PER and CRY proteins start to accumulate, they form PER/CRY heterodimers, which enter the nucleus and negatively feed-back to the CLK/BMAL heterodimers, thus inhibiting their own transcription. The entire process requires about 24 hours, and is efficiently synchronized to the period of the earth rotation by day/night natural cycles and other environmental cues. Mutations and polymorphisms in the clock genes have been implicated in diurnal preference/circadian disorders (26) and are thought to represent a risk factor for the development of sleep-wake disturbances in a number of medical conditions, especially psychiatric disorders (27).

\section{Peripheral clocks and the liver}

In 1972, Langner and co-workers observed that oxygen consumption in a rat liver suspension culture exhibited circadian oscillations (28). This observation paved the way to the demonstration of the existence of circadian clocks other than the master SCN one. These clocks are referred to as 'peripheral', and they are present in virtually every organ and tissue, including the liver, the muscle, the kidney and the hearth (29). When isolated, these tissues maintain robust circadian rhythms of gene expression, which persist in vitro for several days, indicating that the SCN are not required for shortterm rhythmicity (30). However, the SCN master clock is the only clock directly driven by light and 
plays a hierarchical role, determining both the phase and synchrony of clock gene expression in peripheral clocks. Synchronization of peripheral clock timing occurs via the autonomic nervous system, hormone metabolites and metabolic pathways. In addition, cues such as feeding patterns, temperature cycles and physical activity can also reset/phase-shift peripheral oscillators (24). It is believed that peripheral clocks are crucial in regulating specific physiological and metabolic features of most tissues, such as energy metabolism and nutrients processing/storage. For example, disruption of the liver clock results in abnormal glucose and elevation of serum liver enzymes (31). Nonetheless, the molecular links between the metabolic rhythms produced by feeding/fasting, and the resetting of peripheral clocks have represented a puzzle for several years. Recently, it has been demonstrated that metabolic cycles can directly cross-talk to the molecular components of the peripheral circadian clock within the mouse liver (32). In more detail, feeding/fasting cycles are able to locally modify some of the molecular features of crucial clock proteins, thus synchronizing the local clock to their periods and uncoupling it from SCN control. Some of the molecules involved have been identified as the NAD-dependent enzymes Sirtuin1 (SIRT1) (33) and the poly ADP-ribose polymerase-1 (PARP-1), which act as NAD sensors and feedback directly to the core of the liver circadian clock. SIRT1 is a histone deacetylase and it induces CLK-mediated chromatin epigenetic modifications which, in turn, inhibit Per and Cry transcriptional activity. PARP-1, which is also involved in chromatin remodelling, adds ADP-ribose molecules to CLK, reducing its binding to the E-boxes and thus modifying the phase of Per and Cry transcription. Chromatin remodelling is fundamental for the control of a large number of nuclear processes. The above mentioned findings have connected cellular metabolism, epigenetic status, and the circadian clock (34). Finally, an emerging mechanism contributing to the fine tuning of circadian rhythmicity within the liver clock involves specific miRNAs, which have been shown to downregulate or silence circadian genes expression (35).

\section{Homeostatic sleep-wake regulation}


Homeostatic regulation is responsible for the progressive increase in sleep propensity during the waking hours, and its dissipation during sleep. The pioneering studies of Blake and Gerard showed that both the arousal threshold and the dominance of slow electroencephalographic waves are high in the initial part of night sleep, and progressively decrease (36). This was confirmed in subsequent studies, and it was also demonstrated that sleep deprivation produces an increase in slow-wave activity in the following 'recovery' night. By contrast, a daytime nap attenuates slow-wave activity in the subsequent sleep episode (37). These findings indicate that slow-wave activity reliably reflects prior history of sleep and wake. The exact neurochemical correlates of human sleep homeostasis remain unknown, but there is evidence that adenosinergic neurotransmission might play a crucial role (38).

\section{Sleep-wake regulation in patients with cirrhosis}

\section{Circadian sleep-wake regulation}

As melatonin is metabolised in the liver, it has generally been assumed that in patients with cirrhosis its clearance would be delayed, or somehow compromised. There is evidence to support this contention, as patients with cirrhosis show high daytime melatonin levels $(39,40)$, low urinary aMT6s (41), and a reduction in the clearance of exogenously administered melatonin (42). In a study where plasma melatonin and urinary aMT6s were assessed simultaneously over a 36-hour period in strictly controlled laboratory conditions, 24-hour melatonin clearance was comparable to that of healthy controls, while overnight melatonin clearance was reduced (43). In the same and other studies, correlations were observed between the delay in the timing of melatonin/aMT6s peaks and the degree of hepatic failure (9,43-45). However, other circadian abnormalities have also been described, such as delays in the nocturnal rise of melatonin and in its time to peak $(39,43,45)$, which suggest dysfunction of the central SCN circadian clock. The functional integrity of the retinal-hypothalamic axis, and thus of the SCN clock, can be assessed by measuring light-induced 'melatonin suppression' [i.e. the rapid decrease in melatonin levels in response to ocular light exposure at night] and/or by measuring the 24-hour profile 
of at least two variables out of melatonin, cortisol and core body temperature. Montagnese and coworkers demonstrated parallel delays in the onset of melatonin and cortisol rhythms, and attenuated melatonin sensitivity to light in a group of 20 patients with cirrhosis, supporting the hypothesis of central circadian dysfunction $(43,46)$. Other authors $(45)$ reported normal cortisol rhythms in patients with cirrhosis but the number of timed samples was possibly smaller than that required for accurate estimates of rhythm timing, and the confounding effect of light was not controlled for. In the study by Montagnese and co-workers, melatonin sensitivity to light at night was inversely correlated with the timing of the melatonin peak (43), supporting Steindl's original hypothesis that the observed delays in the 24-hour melatonin profile depend on a dysfunctional retinal-hypothalamic axis $(39,47)$.

Some attempt has been made to correlate the changes in the endogenous melatonin rhythm with the sleep disturbances observed in patients with cirrhosis, but the findings have been inconclusive $(39,43)$. It has been suggested that circadian rhythm delays may be associated with delayed sleep habits, although not necessarily with impaired sleep quality alone (9). The combination of evening preference, delayed sleep habits, impaired sleep quality and delayed circadian rhythms is reminiscent of delayed sleep phase syndrome (DSPS), a circadian rhythm sleep disorder characterised by considerable delays in sleep onset/wake times. The goal of treatment for DSPS is to resynchronise the circadian clock with the 24-hour light/dark cycle, and the advancing effect of exposure to bright light in the morning has been shown to be beneficial (48). One encouraging case report suggests that treatment with morning light might be effective in managing the DSPS-like syndrome observed in patients with cirrhosis (49).

\section{Homeostatic sleep-wake regulation}

Less information is available on sleep homeostasis in patients with cirrhosis. Polysomnography has been performed in a limited number of studies, mostly with the aim of evaluating indices of HE rather than sleep homeostatic indices per se. Correlations have been established between the clinical severity 
of encephalopathy and ammonia levels on one hand, and the degree of disruption of sleep architecture on the other $(50,20)$. More recently, nap polysomnography was obtained in a group of patients with cirrhosis and matched controls, at baseline and after the induction of mild hyperammonaemia (18). Hyperammonaemia resulted in increased subjective sleepiness in both groups. However, it led to longer and deeper sleep in healthy volunteers and to shorter, more superficial sleep in cirrhotic patients, pointing to a reduced ability to produce restorative sleep (18). Finally, decreased density of the adenosine receptor A1AR has been described in both cortical and sub-cortical regions of the brain of cirrhotic patients (51), also implicating impaired homeostatic regulation in their sleep-wake abnormalities.

A model for the interaction and the effects of circadian and homeostatic dysfunction in cirrhosis is presented in Figure 1. This is a simple model based on the current available evidence. However, there are multiple, mostly unexplored mechanisms through which cirrhosis and its complications might affect sleep-wake patterns and circadian regulation; these are presented in Figure 2.

\section{Treatment of sleep-wake disturbance in patients with cirrhosis}

Limited data are available on how we should treat sleep-wake disturbances in patients with cirrhosis. This is because sleep-wake assessment is not part of routine hepatological practice, the understanding of the pathophysiology is limited, and these patients are fairly sensitive to psychoactive medication. Even when an 'aetiological' treatment was attempted by Spahr and colleagues, who administered the histamine $\mathrm{H} 1$ blocker hydroxyzine to patients with minimal HE and sleep impairment, some risk of precipitating severe HE was observed (10). The combination of the above issues has resulted in underdiagnosis and cautious, aspecific and potentially inappropriate management. The data currently available allow formulation of the following, simple recommendations: 
i) Routine assessment of sleep quality, sleep timing and daytime sleepiness should be performed (52).

ii) The detection of excessive daytime sleepiness should prompt search for and treatment of hyperammonaemia and HE (17).

iii) Sleep and light hygiene practices should be encouraged, to include regular sleep-wake schedules, exposure to bright light in the early hours of the morning and avoidance of bright light exposure in the evening (DSPS-like treatment). Given the observed impairment in overnight melatonin clearance, the benefits and risks of melatonin administration are worthy of specific, formal studies.

iv) Hypnotics should be used with caution, especially in decompensated patients. No formal trial data are available. However, it is reasonable to assume that the hypnotic of choice should have: negligible hepatic metabolism, short half-life, no active metabolites and limited lipophilia, to avoid prolonged intra-cerebral action. These features should not limit its usefulness, as difficulties falling asleep are considerably more common than early awakening in this patient population.

However, it is only further elucidation of the pathophysiology of sleep-wake abnormalities in patients with cirrhosis that may lead, in time, to the development of specific treatment strategies. Over the last few decades, chronobiologists and sleep scientists have taken an interest in the liver, and they have been rewarded with remarkable discoveries. Hepatologists should try to reciprocate, both for the sake of their patients and their research: there is a liver clock, and it may soon be liver time. 


\section{Funding}

Part of this work was funded by a grant from the Italian Ministry of Health to SM (Giovani Ricercatori 2009). DJS is a Royal Society Wolfson Research Merit Award holder. Stockgrand Ltd. (UK) undertook the melatonin, urinary 6-sulphatoxymelatonin and cortisol assays. Derungs-Waldmann Illuminotecnica (Italy) provided lighting and light measurement equipment.

\section{Acknowledgements}

We are very grateful to all the colleagues, patients and healthy volunteers who, over the years, have helped us with intense research studies both in the UK and Italy. They were cheerful and enthusiastic companions during these sleepless adventures. In particular, we are indebted to Dr. Marsha Morgan, of University College London, for her encouragement and her ability to separate hard information from confounding and noise. 


\section{Legends to Figures}

Figure 1. Cirrhosis-associated abnormalities in the two-process model of sleep regulation. A. Normal interaction between the circadian oscillation in sleep propensity and the increase in homeostatic sleep pressure during the waking hours: the greater the distance between the two curves (23:00), the higher the sleep propensity (22). B. Abnormal interaction between the homeostatic regulation (black line) and the delayed circadian rhythm (red dotted line) in a patient with cirrhosis; grey line: reference circadian oscillation in the healthy population. The lack of synchrony between the two processes leads to a jet-lag East/DSPS-type sleep disorder, which could contribute to the observed difficulties in commencing (increased latency) and maintaining sleep (fragmentation). C. Abnormal interaction between blunted homeostatic fluctuations (red broken line) and shifted/delayed circadian rhythm (red dotted line) in a patient with cirrhosis and HE; grey lines: reference circadian oscillation and homeostatic build-up in the healthy population. Hyperammonaemia/HE-related daytime somnolence may compromise the homeostatic build-up of sleep pressure during the daytime hours, thus contributing to a reduction in slow-wave, restorative sleep.

Figure 2. Hypothetical, largely unexplored pathways through which cirrhosis and its complications may lead to sleep-wake and circadian rhythm abnormalities: A. In compensated cirrhosis, subverted liver architecture/vascularisation may lead to dysfunction of the hepatic clock. In addition, the reduced ability of the liver to store glycogen and synthesise protein may impinge on the physiological, rhythmic changes in glucose/protein metabolism which anticipate and follow meals. B. In decompensated cirrhosis, sympathetic/parasympathetic imbalance may compromise the autonomic transmission of the circadian SCN clock signal to the periphery. In the same context, the combination of hyperdynamic circulation, peripheral vasodilatation and temperature deregulation may impinge on the vascular mechanisms implicated in sleep preparation/sleep onset. In addition, malnutrition and muscle loss may lead to both reduced exercise and blunted response to exercise cues. C. Finally, the presence of HE per 
se, which reduces alertness, might weaken the wake-sleep cycle and compromise the build up of homeostatic sleep pressure. In addition, abnormalities in ocular or cerebral light/dark perception or interpretation might alter the phase of the central SCN circadian clock.

CNS: Central Nervous System; SCN: Suprachiasmatic nuclei 
1. Partinen M, Hublin C. Epidemiology of sleep disorders. In: Kryger MH, Roth T, Dement WC, eds. Principles and Practice of Sleep Medicine. Fourth ed. Philadelphia: Elsevier Saunders, 2005. 626-647.

2. Sherlock S, Summerskill WH, White LP, Phear EA. Portal-systemic encephalopathy; neurological complications of liver disease. Lancet 1954;267:454-457.

3. Wiltfang J, Nolte W, von Heppe J, Bahn E, Pilz J, Hajak G, et al. Sleep disorders and portalsystemic encephalopathy following transjugular intrahepatic portosystemic stent shunt in patients with liver cirrhosis. Relation to plasma tryptophan. Adv Exp Med Biol 1999;467:169176.

4. Bergonzi P, Bianco A, Mazza S, Mennuni G, Zolo P. [Organization of nocturnal sleep in patients with liver cirrhosis: changes before and after treatment with levodopa and lactulose]. Riv Neurol 1975;45:118-123.

5. Cordoba J, Cabrera J, Lataif L, Penev P, Zee P, Blei AT. High prevalence of sleep disturbance in cirrhosis. Hepatology 1998;27:339-345.

6. Bianchi G, Marchesini G, Nicolino F, Graziani R, Sgarbi D, Loguercio C, et al. Psychological status and depression in patients with liver cirrhosis. Dig Liver Dis 2005;37:593-600.

7. Montagnese S, Middleton B, Skene DJ, Morgan MY. Night-time sleep disturbance does not correlate with neuropsychiatric impairment in patients with cirrhosis. Liver Int 2009;29:13721382.

8. Mostacci B, Ferlisi M, Baldi AA, Sama C, Morelli C, Mondini S, et al. Sleep disturbance and daytime sleepiness in patients with cirrhosis: a case control study. Neurol Sci 2008;29:237-240.

9. Montagnese S, Middleton B, Mani AR, Skene DJ, Morgan MY. Sleep and circadian abnormalities in patients with cirrhosis: features of delayed sleep phase syndrome? Metab Brain Dis 2009;24:427-439.

10. Spahr L, Coeytaux A, Giostra E, Hadengue A, Annoni JM. Histamine H1 blocker hydroxyzine improves sleep in patients with cirrhosis and minimal hepatic encephalopathy: a randomized controlled pilot trial. Am J Gastroenterol 2007;102:744-753.

11. Cordoba J, Dupuis J, Gottstein J, Blei AT. Stenosis of a portacaval anastomosis affects circadian locomotor activity in the rat: a multivariable analysis. Am J Physiol 1997;273:G1218-G1225. 
12. Llansola M, Cantero JL, Hita-Yanez E, Mirones-Maldonado MJ, Piedrafita B, Ahabrach H, et al. Progressive reduction of sleep time and quality in rats with hepatic encephalopathy caused by portacaval shunts. Neuroscience 2012;201:199-208.

13. Coy DL, Mehta R, Zee P, Salchli F, Turek FW, Blei AT. Portal-systemic shunting and the disruption of circadian locomotor activity in the rat. Gastroenterology 1992;103:222-228.

14. Kantermann T, Juda M, Merrow M, Roenneberg T. The human circadian clock's seasonal adjustment is disrupted by daylight saving time. Curr Biol 2007;17:1996-2000.

15. Filipski E, Subramanian P, Carriere J, Guettier C, Barbason H, Levi F. Circadian disruption accelerates liver carcinogenesis in mice. Mutat Res 2009;680:95-105.

16. Samanta J, Dhiman RK, Khatri A, Thumburu KK, Grover S, Duseja A, et al. Correlation between degree and quality of sleep disturbance and the level of neuropsychiatric impairment in patients with liver cirrhosis. Metab Brain Dis 2013, in press.

17. De Rui M, Schiff S, Aprile D, Angeli P, Bombonato G, Bolognesi M, et al. Excessive daytime sleepiness and hepatic encephalopathy: it is worth asking. Metab Brain Dis 2013, in press.

18. Bersagliere A, Raduazzo ID, Nardi M, Schiff S, Gatta A, Amodio P, et al. Induced hyperammonemia may compromise the ability to generate restful sleep in patients with cirrhosis. Hepatology 2012;55:869-878.

19. Bersagliere A, Raduazzo ID, Schiff S, Gatta A, Merkel C, Amodio P, et al. Ammonia-related changes in cerebral electrogenesis in healthy subjects and patients with cirrhosis. Clin Neurophysiol 2013;124:492-496.

20. Bajaj JS, Saeian K, Schubert CM, Franco R, Franco J, Heuman DM. Disruption of sleep architecture in minimal hepatic encephalopathy and ghrelin secretion. Aliment Pharmacol Ther 201;34:103-105.

21. Kappus MR, Leszczyszyn DJ, Moses L, Raman S, Heuman DM, Bajaj JS. Effect of obstructive sleep apnea on the sleep architecture in cirrhosis. J Clin Sleep Med 2013;9:247-251.

22. Borbely AA. A two process model of sleep regulation. Hum Neurobiol 1982;1:195-204.

23. Skene DJ, Papagiannidou E, Hashemi E, Snelling J, Lewis DF, Fernandez M, et al. Contribution of CYP1A2 in the hepatic metabolism of melatonin: studies with isolated microsomal preparations and liver slices. J Pineal Res 2001;31:333-342.

24. Green CB, Takahashi JS, Bass J. The meter of metabolism. Cell 2008;134:728-742. 
25. Albrecht U. Timing to perfection: the biology of central and peripheral circadian clocks. Neuron 2012;74:246-260.

26. Archer SN, Robilliard DL, Skene DJ, Smits M, Williams A, Arendt J, et al. A length polymorphism in the circadian clock gene Per3 is linked to delayed sleep phase syndrome and extreme diurnal preference. Sleep 2003;26:413-415.

27. Serretti A, Benedetti F, Mandelli L, Lorenzi C, Pirovano A, Colombo C, et al. Genetic dissection of psychopathological symptoms: insomnia in mood disorders and CLOCK gene polymorphism. Am J Med Genet B Neuropsychiatr Genet 2003;121:35-38.

28. Langner R, Rensing L. Circadian rhythm of oxygen consumption in rat liver suspension culture: changes of pattern. Z Naturforsch B 1972;27:1117-1118.

29. Balsalobre A, Damiola F, Schibler U. A serum shock induces circadian gene expression in mammalian tissue culture cells. Cell 1998;93:929-937.

30. Yamazaki S, Numano R, Abe M, Hida A, Takahashi R, Ueda M, et al. Resetting central and peripheral circadian oscillators in transgenic rats. Science 2000;288:682-685.

31. Iurisci I, Filipski E, Sallam H, Harper F, Guettier C, Maire I, et al. Liver circadian clock, a pharmacologic target of cyclin-dependent kinase inhibitor seliciclib. Chronobiol Int 2009;26:1169-1188.

32. Asher G, Schibler U. Crosstalk between components of circadian and metabolic cycles in mammals. Cell Metab 2011;13:125-137.

33. Nakahata Y, Sahar S, Astarita G, Kaluzova M, Sassone-Corsi P. Circadian control of the NAD+ salvage pathway by CLOCK-SIRT1. Science 2009;324:654-657.

34. Katada S, Sassone-Corsi P. The histone methyltransferase MLL1 permits the oscillation of circadian gene expression. Nat Struct Mol Biol 2010;17:1414-1421.

35. Gatfield D, Le MG, Vejnar CE, Gerlach D, Schaad O, Fleury-Olela F, et al. Integration of microRNA miR-122 in hepatic circadian gene expression. Genes Dev 2009;23:1313-1326.

36. Blake H, Gerard RW. Brain potentials during sleep. Am J Physiol 1937;119:692-703.

37. Werth E, Dijk DJ, Achermann P, Borbely AA. Dynamics of the sleep EEG after an early evening nap: experimental data and simulations. Am J Physiol 1996;271:R501-R510.

38. Landolt HP. Sleep homeostasis: a role for adenosine in humans? Biochem Pharmacol 2008 Jun $1 ; 75: 2070-2079$. 
39. Steindl PE, Finn B, Bendok B, Rothke S, Zee PC, Blei AT. Disruption of the diurnal rhythm of plasma melatonin in cirrhosis. Ann Intern Med 1995;123:274-277.

40. Chojnacki C, Wachowska-Kelly P, Blasiak J, Reiter RJ, Chojnacki J. Melatonin secretion and metabolism in patients with hepatic encephalopathy. J Gastroenterol Hepatol 2013, in press.

41. Steindl PE, Ferenci P, Marktl W. Impaired hepatic catabolism of melatonin in cirrhosis. Ann Intern Med 1997;127:494.

42. Iguchi H, Kato KI, Ibayashi H. Melatonin serum levels and metabolic clearance rate in patients with liver cirrhosis. J Clin Endocrinol Metab 1982;54:1025-1027.

43. Montagnese S, Middleton B, Mani AR, Skene DJ, Morgan MY. On the origin and the consequences of circadian abnormalities in patients with cirrhosis. Am J Gastroenterol 2010;105:1773-1781.

44. Piscaglia F, Hermida RC, Siringo S, Legnani C, Ramadori G, Bolondi L. Cirrhosis does not shift the circadian phase of plasma fibrinolysis. Am J Gastroenterol 2002;97:1512-1517.

45. Velissaris D, Karanikolas M, Kalogeropoulos A, Solomou E, Polychronopoulos P, Thomopoulos $\mathrm{K}$, et al. Pituitary hormone circadian rhythm alterations in cirrhosis patients with subclinical hepatic encephalopathy. World J Gastroenterol 2008;14:4190-4195.

46. Montagnese S, Middleton B, Mani AR, Skene DJ, Morgan MY. Changes in the 24-h plasma cortisol rhythm in patients with cirrhosis. J Hepatol 2011;54:588-90

47. Blei AT, Zee P. Abnormalities of circadian rhythmicity in liver disease. J Hepatol 1998;29:832835.

48. Weyerbrock A, Timmer J, Hohagen F, Berger M, Bauer J. Effects of light and chronotherapy on human circadian rhythms in delayed sleep phase syndrome: cytokines, cortisol, growth hormone, and the sleep-wake cycle. Biol Psychiatry 1996;40:794-797.

49. De RM, Gaiani S, Middleton B, Skene DJ, Schiff S, Gatta A, et al. Bright times for patients with cirrhosis and delayed sleep habits: a case report on the beneficial effect of light therapy. Am J Gastroenterol 2011;106:2048-2049.

50. Kurtz D, Zenglein JP, Imler M, Girardel M, Grinspan G, Peter B, et al. [Night sleep in portocaval encephalopathy]. Electroencephalogr Clin Neurophysiol 1972;33:167-178.

51. Boy C, Meyer PT, Kircheis G, Holschbach MH, Herzog H, Elmenhorst D, et al. Cerebral A1 adenosine receptors (A1AR) in liver cirrhosis. Eur J Nucl Med Mol Imaging 2008;35:589-597. 
52. Montagnese S, Middleton B, Skene DJ, Morgan MY. Sleep-wake patterns in patients with cirrhosis: all you need to know on a single sheet. A simple sleep questionnaire for clinical use. J Hepatol 2009;51:690-695. 